\title{
Evaluation and prediction of therapeutic response for patients with hepatocellular carcinoma receiving stereotactic body radiotherapy using serial computed tomography scans and radiomics
}

\author{
Yang-Hong Dai ${ }^{1}$, Po-Chien Shen ${ }^{1}$, Ying-Fu Wang ${ }^{1}$, Wei-Chou Chang ${ }^{2}$, Cheng-Hsiang Lo ${ }^{1}, J^{2}$-Fu Yang ${ }^{1}$, \\ Chun-Shu Lin ${ }^{1}$, Hsing-Lung Chao ${ }^{1}$, Chieh-Sheng Lu ${ }^{3}$, Wen-Yen Huang ${ }^{1}$ \\ ${ }^{1}$ Department of Radiation Oncology, Tri-Service General Hospital, National Defense Medical Center, Taiwan; ${ }^{2}$ Department of Radiology, Tri- \\ Service General Hospital, National Defense Medical Center, Taiwan; ${ }^{3}$ Department of Internal Medicine, Kaohsiung Armed Forces General Hospital, \\ Kaohsiung, Taiwan \\ Contributions: (I) Conception and design: YH Dai; (II) Administrative support: WY Huang; (III) Provision of study materials or patients: WY Huang, \\ CH Lo; (IV) Collection and assembly of data: YH Dai, CH Lo; (V) Data analysis and interpretation: YH Dai, PC Shen, YF Wang; (VI) Manuscript \\ writing: All authors; (VII) Final approval of manuscript: All authors. \\ Correspondence to: Wen-Yen Huang, MD. Department of Radiation Oncology, Tri-Service General Hospital, National Defense Medical Center, No. \\ 325, Chengong Rd., Sec. 2, Neihu, Taipei 114, Taiwan. Email: hwyyi@yahoo.com.tw; Chieh-Sheng Lu, MD. Department of Internal Medicine, \\ Kaohsiung Armed Forces General Hospital, Kaohsiung, Taiwan. Email: chengsem@gmail.com.
}

Background: Stereotactic body radiotherapy (SBRT) allows accurate high-energy dose delivery to tumors of interest and has been shown effective for unresectable hepatocellular carcinoma (HCC). In the era of personalized medicine, we aimed to predict therapeutic outcome of SBRT in HCC patients using computed tomography (CT) images and radiomic analyses.

Methods: A total of 77 HCC patients undergoing SBRT were retrospectively analyzed. Five millimeter of peritumoral area was identified using semi-automatic method on $3 \mathrm{D}$ slicer, and overall 839 radiomic features were extracted, followed by selection with elastic net regularization (ENR). Treatment response was evaluated by CT follow-ups and was quantified by mRECIST. Multivariate logistic regression model was trained and the model performance was evaluated by receiver operating characteristic (ROC) curve analysis.

Results: During 4 imaging follow-ups, 34 tumors (43.6\%) achieved response, and 5 tumors had complete response (6.4\%). Among the 34 tumors, most tumors achieved response at first follow-up (FU1) (N=21, 61.7\%). Using logistic regression, we identified that wavelet high-high-lowpass filtering (HHL) GLCM (GLCM ${ }^{\text {waveletHHL}}$ ) was the most significant feature for response at FU1 (coefficient $=0.6805, \mathrm{P}=0.0373,95 \%$ CI, 0.0401-1.3208). With this single feature, logistic regression model was built and the model accuracy was 0.83 (AUC $=0.71,95 \%$ CI, 0.45-0.81). We also observed responders at FU1 had a trend toward higher survival probability within 2 years $(\mathrm{P}=0.16)$.

Conclusions: The therapeutic impact of SBRT in HCC could be addressed by the tumor response at FU1, which corresponded to the local control about 1 year after therapy.

Keywords: Stereotactic body radiotherapy (SBRT); radiotherapy; hepatocellular carcinoma (HCC); radiomics

Received: 21 April 2020. Accepted: 25 August 2020; Published: 30 December 2020.

doi: $10.21037 /$ tro-20-38

View this article at: http://dx.doi.org/10.21037/tro-20-38 


\section{Introduction}

In the era of conformal radiation therapy (RT), stereotactic body radiotherapy (SBRT) allows for higher radiation dose delivery to the target volume with tight conformity. Its steep dose gradient provides a rationale for the treatment of a single tumor while limiting the dose to the adjacent parenchyma (1). In fact, SBRT has been shown effective and well-tolerated in patients with medically inoperable stage I non-small cell lung cancer and oligometastatic prostate cancer $(2,3)$. On the other hand, the evidence for SBRT in hepatocellular carcinoma (HCC) is accumulating. Several prospective trials have shown SBRT is feasible in HCC, with high local control rate ranging from $87 \%$ to $100 \%$ (4-7). Its advantages are further addressed in another recent prospective study, showing safety and effectiveness of SBRT in HCC with or without prior liver-directed therapy (8).

Although high local control rate could be achieved with SBRT in HCC, the majority of published studies reported broadly defined 1-year and 2-year local control rate, overlooking the dynamics of therapeutic response $(7,9,10)$. Bhatt et al. quantified gross tumor volume (GTV) change during SBRT and at the first follow-up, and they found the mean tumor volume showed significant shrinkage at the first follow-up after completion of SBRT (most >100 days) (11). Goyal et al. observed a mean decrease in tumor volume of $60 \%$ at 3 months after SBRT in HCC (12). However, there were only 6 patients in this study and the data of tumor size change during each follow-up remained elusive. Additionally, despite clear etiology, HCC is characterized by intertumor and intratumor heterogeneity, making the development of systemic targeted therapy challenged (13). This difficulty might be overcome with nonspecific highenergy radiation, based on reported high local control rate for SBRT across many heterogeneous prospective and retrospective studies. Even though, there still exist therapeutic discrepancy that could result from different functional liver parameters (14). Therefore, in this context, development of tools to predict treatment response or patient survival is crucial.

Radiomics is a burgeoning field that converts tremendous imaging information into various reproducible features through sophisticated algorithms $(15,16)$. It has been explored in HCC, and demonstrates excellent performance in clinical diagnosis and prognosis (17-19). For radiotherapy, Cozzi et al. adopted radiomics to predict local control and survival in HCC patients and identified high model performance (19). Nevertheless, this study used conventional fraction size and lacked validation cohort.

Several studies showed the peritumoral microenvironment in HCC is associated with recurrence and survival (20-23). Therefore, we aimed to investigate the impact of this region on tumor response using radiomics. In this study, we retrospectively reviewed HCC patients who received SBRT in our hospital. We assessed the tumor size change through serial dynamic computed tomography (CT) scans and aimed to quantify the therapeutic response after SBRT. We then used radiomic approach at the peritumoral region to predict local response during meaningful follow-up period. We present the following article in accordance with the STROBE reporting checklist (available at http://dx.doi. org/10.21037/tro-20-38).

\section{Methods}

\section{Patients}

One hundred and fifty-one HCC patients who received SBRT at our hospital between 2007 and 2016 were retrospectively reviewed. Eligible patients should have at least one set of follow-up CT scan or magnetic resonance image (MRI) after therapy for treatment response evaluation. For HCC with Barcelona Clinic Liver Cancer (BCLC) stage A to C, radiotherapy was considered eligible and those patients were included in our study. Moreover, patients who had undergone prior local therapy like transarterial chemoembolization (TACE) and radiofrequency ablation (RFA) were also enrolled. Because of the retrospective nature of this study, we have obtained approval from Institutional Review Board for a waiver of informed consent (IRB number: 1-107-05-016). This study was conducted in accordance with the Declaration of Helsinki (as revised in 2013).

\section{SBRT and clinical endpoints}

All patients were treated with SBRT using the Cyberknife image-guided radiosurgery system (Accuray, USA) as described previously $(9,24)$. Therapeutic response was evaluated using modified response evaluation criteria in solid tumors (mRECIST), which is based on the measurement of viable tumor during arterial phase in contrast-enhanced CT $(25,26)$. By measuring the size of manually contoured GTV, initial tumor size was defined and was set as the baseline. The follow-up images, either CT or MRI, were reviewed by one experienced radiologist and the tumor sizes were 


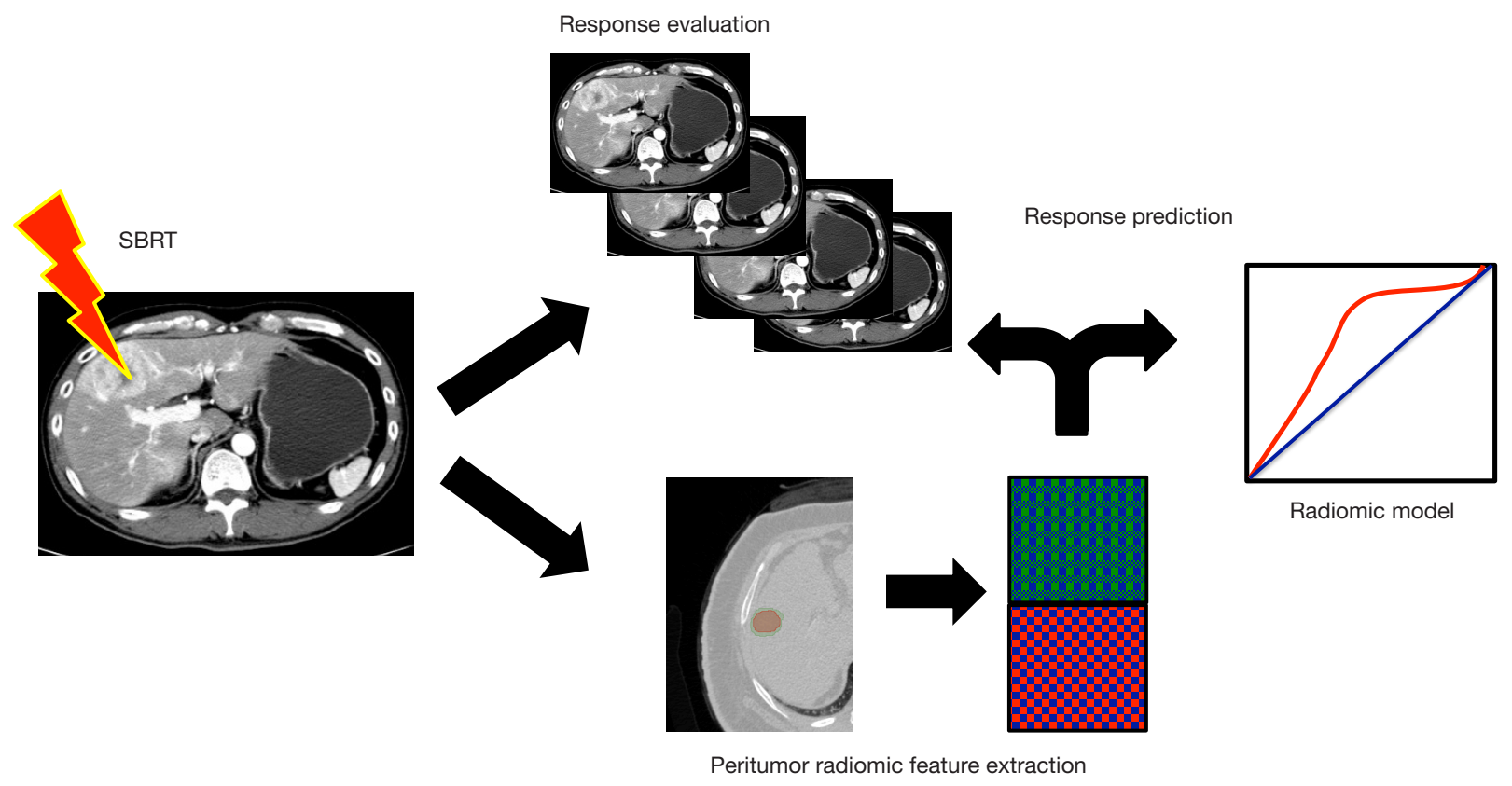

Figure 1 Radiomic analysis workflow.

compared to the baseline for calculation of serial changes of mRECIST. Irradiated tumors achieving partial $(>30 \%$ decrease in longest diameter) and complete responses were considered responders. On the contrary, progressively increased tumor diameter $>20 \%$ inside radiation field, as per mRECIST criteria, was considered in-field failure (IFF). As for the therapeutic aspect, we aimed to know the effect of radiation dose on the tumor response. For varying dose fractionations, equivalent dose in 2 gray (Gy) per fraction (EQD2) was used to account for similar biological basis. Additionally, radiation-induced liver disease (RILD) was reviewed for each patient after SBRT.

\section{Extraction of radiomic features}

We aimed to investigate the impact of peritumoral region on tumor response using radiomics. Each HCC patient received dynamic CT scan with contrast prior to SBRT for simulation. All scans were performed with a tube voltage of $120 \mathrm{kVp}$ and a pitch of 0.984 . The GTV was contoured on the CT slices in the arterial phase. The treatment planning in the digital imaging and communication in medicine-RT (DICOM-RT) format was extracted and imported into 3D slicer (www.slicer.org). The DICOMRT contains detailed information about radiation dose and RT structures including previously contoured GTV.
The module of 'SlicerRT' and 'Radiomics' were adopted for analyses. Briefly, each CT scans were standardized first by isotropic spacing $\left(0.68 \times 0.68 \times 0.68 \mathrm{~mm}^{3}\right.$ for each voxel). Semi-automatic margin expansion from the GTV was then done in $3 \mathrm{D}$ slicer. The original tumor volume was subtracted and a $5 \mathrm{~mm}$ peritumoral ring was left for further radiomic analysis. Large vessels, adjacent structures and air were excluded. Hounsfield units were discretized by 400 discrete values, resulting in a bin width of 25 units. Radiomic features were extracted using 'pyradiomics' package implemented in the 'Radiomics' module in $3 \mathrm{D}$ slicer. The features included first order statistics, shape, and four categories of grey-level matrices calculated in three dimensions with and without wavelet transformation, leading to a total of 839 features. Values of extracted features were normalized with a final range between 0 and 1 . The radiomic workflow was shown in Figure 1.

\section{Feature selection}

To obtain key features for therapeutic response evaluation, elastic net regularization (ENR) was used. ENR is advantageous in selecting the best set of features while minimizing the residual sum of squares of estimating errors (27). With added penalty term, ENR achieves tradeoff between fitting performance and model complexity, 
reducing over-fitting or bias. This is suitable for regression problems and has been widely used in models built from radiomics. We used $\lambda=0.5$ and loop iteration to identify the optimal $\alpha$ between 0 and 1 with 10 -fold cross-validation. Features with non-zero coefficients were considered predictive and were selected.

\section{Construction of predictive model}

The predictive value of the selected radiomic features was assessed with multivariate logistic regression model. The primary cohort was separated into training and testing cohorts with a ratio of 7:3. The training cohort was used for model construction and the model performance was illustrated using receiver operating characteristic (ROC) curve analysis. The training samples were bootstrapped for 1,000 times to calculate the area under curve (AUC), sensitivity and specificity with $95 \%$ CI. The regression model was then applied to the testing cohort to evaluate the accuracy of prediction.

\section{Statistical analysis}

Differences in distribution of clinical variables between responders and non-responders were assessed with the Fisher exact test. These variables included age, sex, BCLC stage, Child-Pugh score (CP), portal vein thrombosis (PVT), viral status, previous TACE, albumin-bilirubin (ALBI) score and $\alpha$-fetoprotein (AFP). Wilcoxon and Kruskal-Wallis tests were used to evaluate the unpaired difference between two groups and variance among multiple groups, respectively. Pearson's and Spearman's coefficients were calculated to identify the correlation of size change between imaging follow-ups. Kaplan-Meier analysis and log-rank test were performed to compare overall survival between responders and non-responders. All statistical analyses were conducted with Spyder (Python version 3.6) and $\mathrm{R}$ software ( $\mathrm{R}$ version 1.1.383). $\mathrm{P}<0.05$ was considered statistically significant.

\section{Results}

\section{Patients}

After excluding ineligible patients with no follow-up CT images, a total of 77 patients and 78 tumors were analyzed. The patient characteristics were summarized in Table 1 . The median follow up time was 11.27 months, with a median imaging follow-up of 3 visits (range, 1-9). The average post-
SBRT imaging follow-ups time were $3.12 \pm 1.98,7.43 \pm 3.86$, $12.70 \pm 5.34,17.51 \pm 9.75$ months (Table 1). About half of the patients received 3 times of imaging acquisition (42.8\%), and only 14 patients and 7 patients $(18.1 \%$ and $9.1 \%)$ had the fourth and fifth follow-up images. The missing information was caused by patient death or loss of followup at our hospital. Therefore, our study focused on the first 4 times of imaging follow-ups to assess the therapeutic response (FU1, FU2, FU3 and FU4).

\section{Therapeutic response analysis}

During 4 imaging follow-ups, 34 tumors (43.6\%) achieved response, and 5 tumors had complete response (6.4\%). Among the 34 tumors, most tumors achieved response at FU1 $(\mathrm{N}=21,61.7 \%) ; 8(23.5 \%)$ and $3(8.8 \%)$ tumors achieved response at FU2 and FU3, respectively (Figure $2 A$, Table 1). However, no tumor achieved response at FU4. Local control was $97.4 \%, 93.2 \%, 94.1 \%$ and $81.3 \%$ at FU1, $\mathrm{FU} 2, \mathrm{FU} 3$ and FU4. For those having response at FU1, 3 tumors $(3 / 21,14.3 \%)$ developed IFF later; and for tumors showing response at FU2 and FU3, $2(2 / 8,25 \%)$ and $1(1 / 3$, $33.3 \%)$ tumor developed IFF afterwards. Furthermore, responders at FU1 paralleled response at FU3 (100\%), and only 9 non-responders at FU1 achieved response at FU3 $(15.5 \%)$.

All tumor responses were normalized by dividing the change in tumor sizes, as defined by mRECIST, with baseline tumor sizes. The serial responses were illustrated in Figure 2B. From the parallel plot, we noticed relatively distinct tumor response at FU1 (mean response $=-0.179 \pm 0.275)$. However, there were no significant differences among the four follow-up times $(-0.046 \pm 0.267$, $-0.1227 \pm 0.221$ and $0.089 \pm 0.216$, for FU2, FU3 and FU4, $\mathrm{P}=0.237$ ) (Figure 2C). The correlation between response at $\mathrm{FU} 1$ and the subsequent responses was highest for the FU2 and FU3 (Table 2). These results indicated that the response evaluated at FU1 had moderate positive correlation with the subsequent responses, which could help address the local control with SBRT within one year.

We then therefore focused on the response at FU1. Cumulative responses from FU1 to FU4 for responders and non-responders at FU1 were assessed, and responders at FU1 showed apparent left shift toward the responsive region, compared to the non-responders (Figure 2D). This plot visualized a higher overall response for responder at FU1. To investigate whether the clinical variables had 
Table 1 Characteristics of patient data

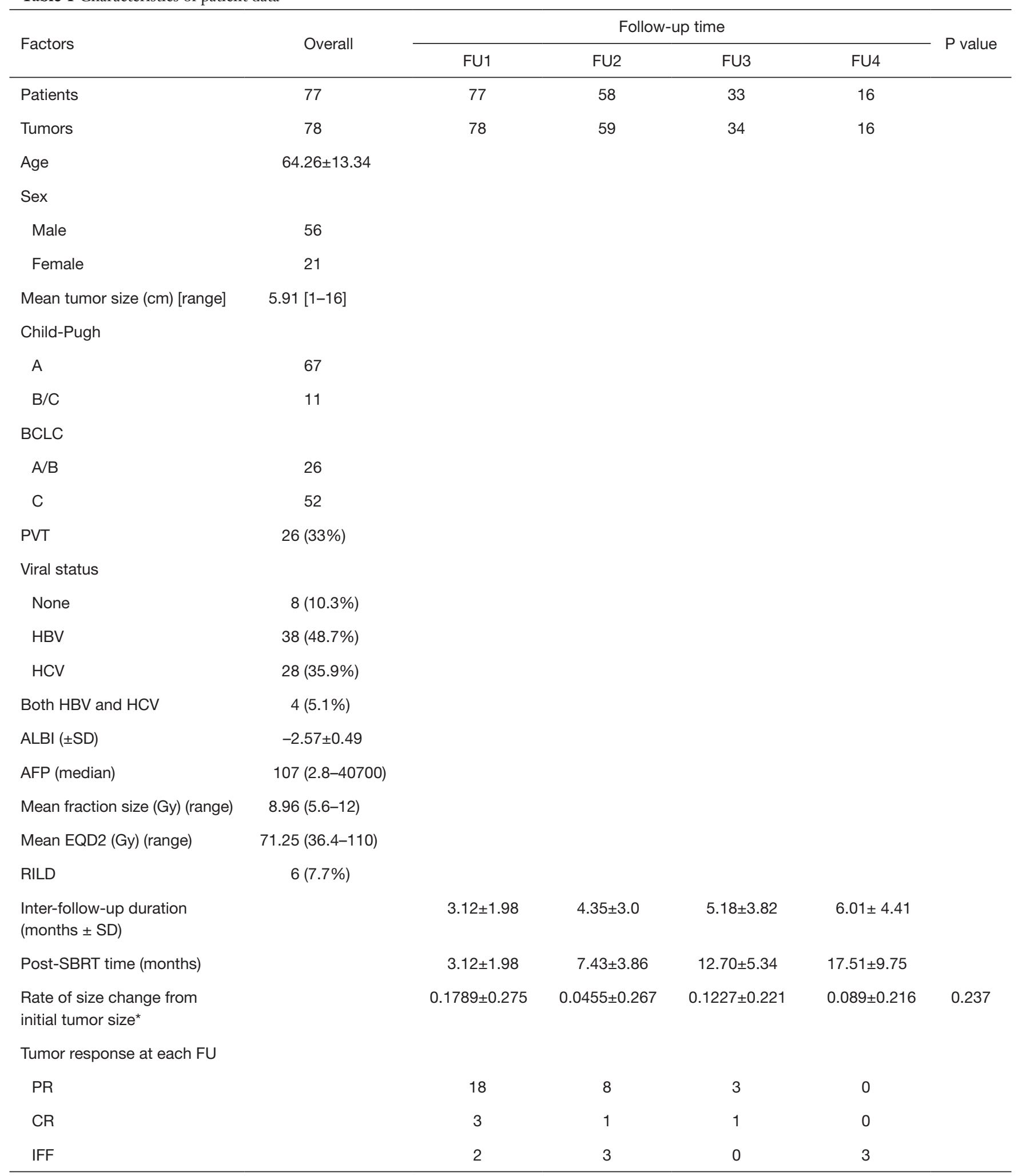

Table 1 (continued) 
Table 1 (continued)

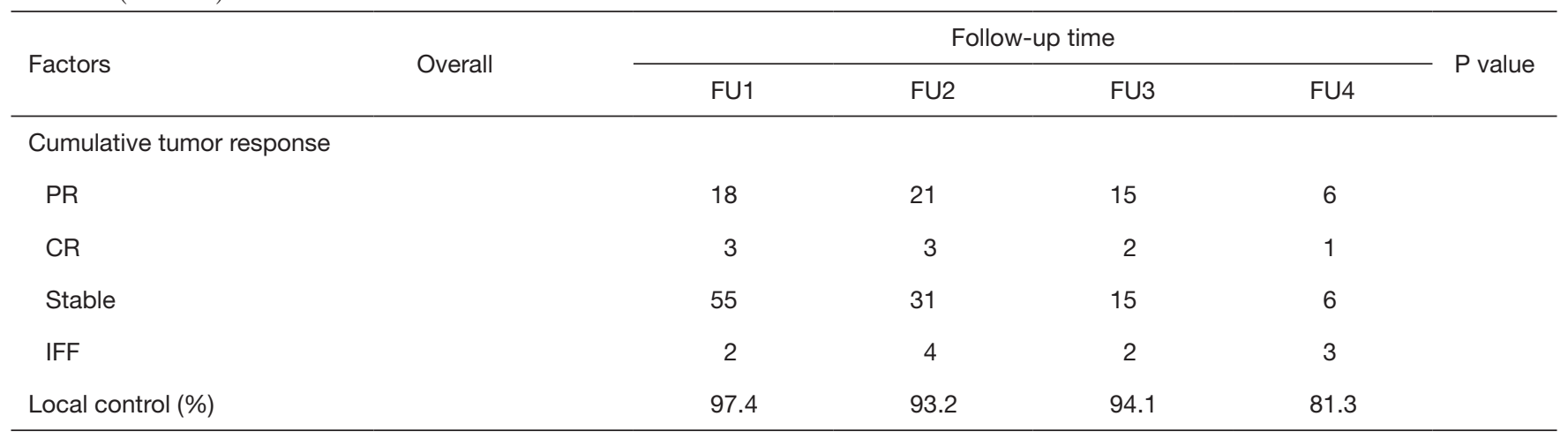

*, Defined by mRECIST (modified response evaluation criteria in solid tumor). FU, follow-up; CP, Child-Pugh; PVT, portal vein thrombosis; ECOG, Eastern Cooperative Oncology Group; ALBI, albumin-bilirubin; AFP, $\alpha$-fetoprotein; Gy, gray; RILD, radiation-induced liver disease; SD, standard deviation; SBRT, stereotactic body radiotherapy; PR, partial response; CR, complete response; IFF, in-field failure.

A
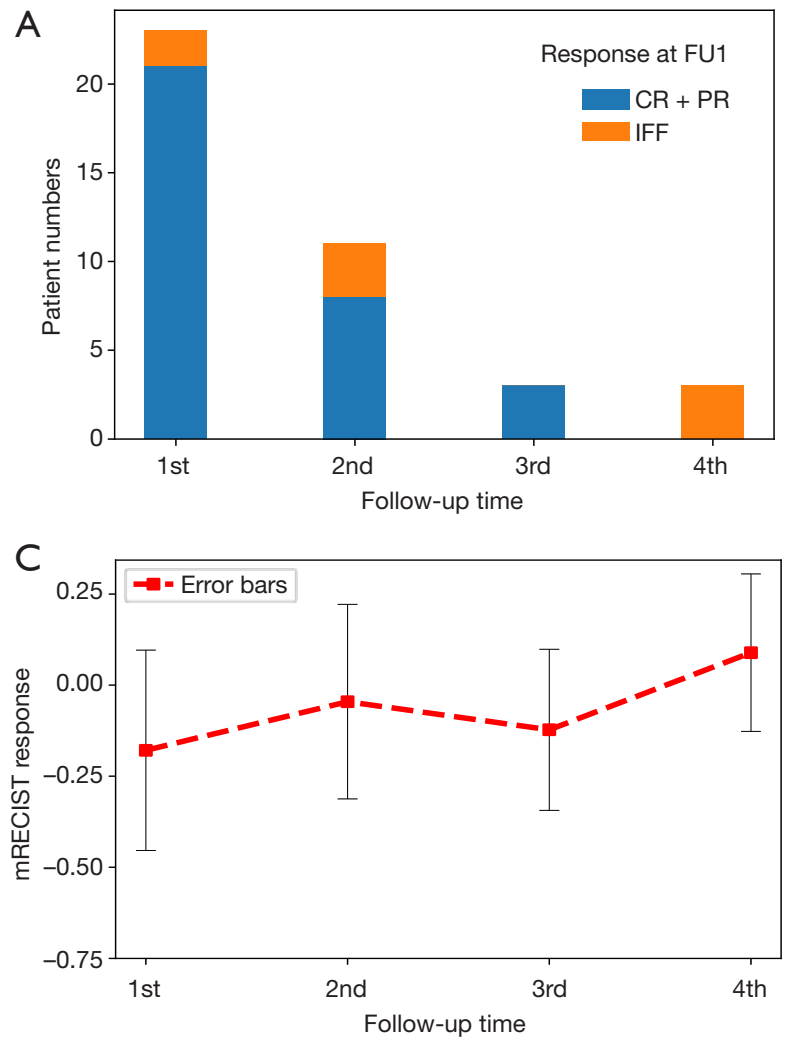

B

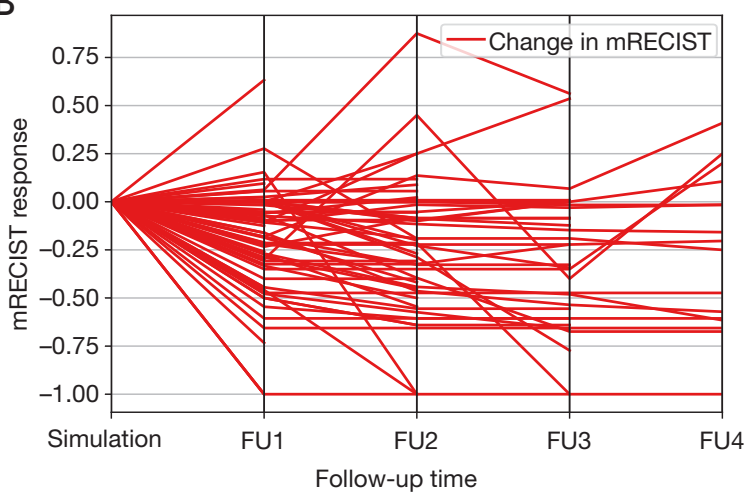

D

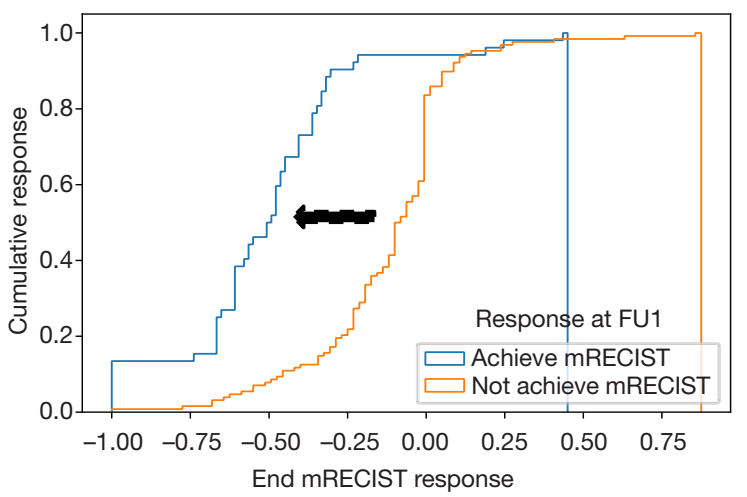

Figure 2 Therapeutic response during four follow-ups. (A) Respective patient numbers for responder and non-responder. (B) Parallel plot of mRECIST for the four follow-ups. The Y-axis indicated the tumor response at each follow-up time according to mRECIST. Each mRECIST was defined as the response at that time point relative the tumor size observed initially. (C) Relative response for the four follow-ups. Each tumor size on the follow-up images was normalized to the baseline pre-treatment tumor size. Each point represented the mean tumor size changes relative to the tumor sizes at the previous follow-up time. (D) Cumulative response between responder and nonresponder. The $y$-axis indicated the final mRECIST response achieved in responders or non-responders at FU1, respectively. The $\mathrm{x}$-axis represented the mRECIST response at FU1. The black arrow indicated left-shift, suggesting an overall higher portion of responsive tumors. 
Table 2 Correlation of FU1 with FU2, FU3 and FU4

\begin{tabular}{lcccc}
\hline & Pearson & P value & Spearman & P value \\
\hline FU2 & 0.631 & $<0.001$ & 0.679 & $<0.001$ \\
FU3 & 0.623 & $<0.001$ & 0.643 & $<0.001$ \\
FU4 & 0.44 & 0.085 & 0.31 & 0.249 \\
\hline
\end{tabular}

FU, follow-up.

Table 3 Factors associated with response at FU1

\begin{tabular}{|c|c|c|c|}
\hline Clinical factors & Responder & Non responder & $P$ value \\
\hline Sex & & & 0.066 \\
\hline Male & 13 & 43 & \\
\hline Female & 9 & 13 & \\
\hline Age & & & 0.078 \\
\hline$<60$ & 6 & 25 & \\
\hline$\geq 60$ & 16 & 31 & \\
\hline Viral status & & & 0.217 \\
\hline Viral infection & 21 & 49 & \\
\hline None & 1 & 7 & \\
\hline $\mathrm{CP}$ & & & ${ }^{\star} 0.023$ \\
\hline A & 22 & 46 & \\
\hline $\mathrm{B} / \mathrm{C}$ & 0 & 10 & \\
\hline BCLC & & & 0.121 \\
\hline $\mathrm{A} / \mathrm{B}$ & 9 & 16 & \\
\hline $\mathrm{C}$ & 13 & 40 & \\
\hline Pre-SBRT TACE & & & 0.062 \\
\hline Yes & 9 & 33 & \\
\hline None & 13 & 23 & \\
\hline AFP & & & 0.233 \\
\hline$<20$ & 6 & 14 & \\
\hline$\geq 20$ & 16 & 42 & \\
\hline ALBI & & & 0.076 \\
\hline Grade 1 & 13 & 25 & \\
\hline Grade 2 & 9 & 31 & \\
\hline
\end{tabular}

BCLC, Barcelona Clinic Liver Cancer; SBRT, stereotactic body radiotherapy; TACE, transarterial chemoembolization; AFP, $\alpha$-fetoprotein; ALBI, albumin-bilirubin. impact on the response at FU1, Fisher exact test was performed and we found there were no differences of distribution between responder and non-responders for factors such as age, sex, viral status, prior TACE, BCLC stages, ALBI and AFP (Table 3) (all P>0.05). However, patients with $\mathrm{CP} \mathrm{B} / \mathrm{C}$ showed no response at $\mathrm{FU} 1(\mathrm{P}=0.023)$. In terms of toxicity, a total of 6 patients (7.8\%) experienced RILD. 3 of them had classical RILD, while the other 3 patients had non-classical RILD (Table 1).

\section{The effect of radiation dose on response at FU1}

The effect of EQD2 on tumor response was shown in Figure 3. The mean tumor responses were $-0.165 \pm 0.233$, $-0.144 \pm 0.273$ and $-0.225 \pm 0.307$, for $30-60$ Gy (Low), 60-80 Gy (Intermediate) and $>80$ Gy (High), respectively $(\mathrm{P}=0.2661)$ (Figure $3 A)$. EQD2 was further divided by tumor size and the results were categorized into 'Norm-Low' ( $\mathrm{N}=26,2.5-9.54)$, 'Norm-Intermediate' ( $\mathrm{N}=26,9.69-20.13$ ) and 'Norm-High' ( $\mathrm{N}=26,20.34-71.2)$, corresponding to the mean tumor responses of $-0.156 \pm 0.203,-0.147 \pm 0.381$, and $-0.233 \pm 0.298(\mathrm{P}=0.651)$ (Figure $3 B)$. The EQD2 and respective average tumor size were summarized in Table 4 . The serial response for the three groups was shown in Figure 3. Although there was no significant difference among the three groups at each follow-up (Figure $3 C$, all, $\mathrm{P}>0.05$ ), tumors in the 'Norm-High' group had more responders, as compared to the 'Norm-Low' group at FU1 $(\mathrm{P}=0.002)$ (Table 5). Of note, two tumors showing in-field progression at FU1 were in the 'Norm-Intermediate' group.

Among patients with $\mathrm{CP} B / \mathrm{C}(\mathrm{N}=11), 10$ tumors (10/11, 90.9\%) were in the 'Norm-Low' and 'Norm-Intermediate' groups, suggesting relatively lower dose were prescribed in these patients.

\section{Construction of predictive model for response at FU1}

Here we aimed to use radiomics to construct the predictive 

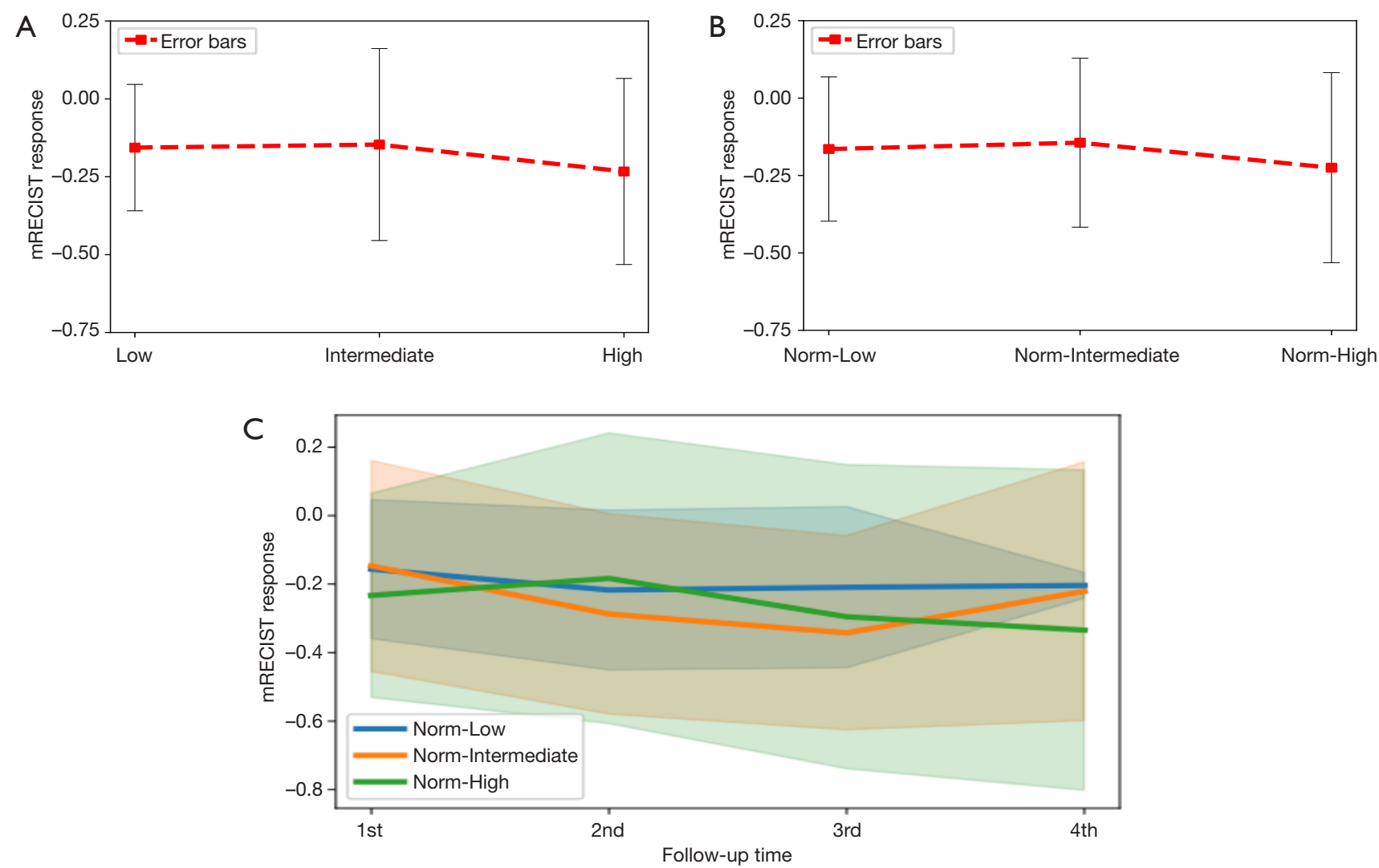

Figure 3 Tumor response according to radiation dose. (A) Tumor response for low EQD2 (30-60 Gy), intermediate EQD2 (60-80 Gy) and high EQD2 (>80 Gy). (B) Tumor response for EQD2 normalized by tumor size. (C) Serial tumor response defined by mRECIST during the four follow-ups.

Table 4 Radiation dose and tumor size in different groups

\begin{tabular}{llc}
\hline & Radiation dose (mean) (Gy) & Tumor size (mean) (cm) \\
\hline EQD2 & & $6.98 \pm 4.16$ \\
30-60 Gy (Low) & $51.68 \pm 8.07$ & $6.46 \pm 3.37$ \\
60-80 Gy (Intermediate) & $70.48 \pm 3.63$ & $4.37 \pm 3.15$ \\
$>80$ Gy (High) & $90.83 \pm 9.64$ & $9.63 \pm 2.99$ \\
EQD2 for normalization & & $5.61 \pm 1.55$ \\
Norm-Low & $58.08 \pm 11.97$ & $2.48 \pm 0.95$ \\
Norm-Intermediate & $73.89 \pm 12.68$ & $81.79 \pm 18.81$ \\
Norm-High & & \\
\hline
\end{tabular}

EQD2, equivalent dose in 2 gray (Gy); cm, centimeter.

model for response at FU1. Among the 839 features, 6 features with non-zero coefficients were identified by ENR to be of predictive value (Table 6). All of these features were based on the wavelet transformation. Using logistic regression, we identified that wavelet high -high-lowpass filtering (HHL) GLCM (GLCM ${ }^{\text {waveletHHL }}$ ) was the most significant feature (coefficient $=0.6805, \mathrm{P}=0.0373,95 \%$ CI, 0.0401-1.3208), and there was significant difference 
Table 5 Distribution of response between 'Norm-Low' and 'Norm-High' groups

\begin{tabular}{|c|c|c|c|}
\hline Normalized EQD2 & \multicolumn{2}{|c|}{ Response } & $P$ value \\
\hline FU1 & & & ${ }^{*} 0.002$ \\
\hline Low & 4 & 21 & \\
\hline High & 10 & 16 & \\
\hline Low & 8 & 11 & \\
\hline High & 9 & 12 & \\
\hline FU3 & & & 0.092 \\
\hline Low & 2 & 7 & \\
\hline Low & 0 & 3 & \\
\hline High & 4 & 7 & \\
\hline
\end{tabular}

*, $P<0.05$. EQD2, equivalent dose in 2 gray.

of feature value between responders and non-responders (Figure $4 A)(\mathrm{P}=0.0012)$. With this single feature, logistic regression model was built and the model accuracy in the testing cohort was $0.83(95 \% \mathrm{CI}=0.81-0.86$; $\mathrm{AUC}=0.71$, 95\% CI, 0.45-0.81) (Figure 4B). In multivariate logistic regression model, the higher model accuracy was achieved (0.88, AUC $=0.75,95 \%$ CI, 0.5-0.833) (Figure 4C).

\section{Survival analysis}

Kaplan-Meier analysis was performed to see whether tumors achieving response at FU1 could affect the survival outcome. Even though the median survival was not reached in both groups, we observed responders at FU1 had a trend toward higher survival probability within 2 years $(\mathrm{P}=0.16)$ (Figure 5).

\section{Discussion}

In this study, we used serial imaging follow-ups to investigate the tumor response after SBRT in HCC. We found most tumors $(\mathrm{N}=21)$ achieved at least partial response at $\mathrm{FU} 1$ $(3.12 \pm 1.98$ months), and these tumors maintained response at later follow-ups ( $\mathrm{N}=21$ at $\mathrm{FU} 3,12.70 \pm 5.34$ months). However, the correlation of response at FU1 decreased when the follow-up time prolonged till FU4. This led
Table 6 Selected features for local response at FU1

\begin{tabular}{ll}
\hline Selected features & Values \\
\hline Wavelet first order & \\
Mean & -0.28591 \\
Wavelet HLH & \\
Correlation & 0.217876 \\
Median & -0.2025 \\
Wavelet HHL & \\
Interquartile range & -0.22081 \\
GLCM & -0.0001097 \\
GLSZM & -0.20219
\end{tabular}

HLH, high-low-highpass filter; HHL, high-high-lowpass filter; GLCM, grey-level co-occurrence matrix; GLSZM, grey level.

to the local control rate of $94.2 \%$ at the first year and $81.3 \%$ at about 17 months, which was supported by our previous study, showing high local control rate of $87.6 \%$ and $75.1 \%$ at 1 - and 2-year, respectively (9). Based on the moderate high correlation of FU1 with FU2 and FU3, and no difference in response at each follow-up, we suggested that the tumor response at 1 year could be reflected by the response at FU1. 

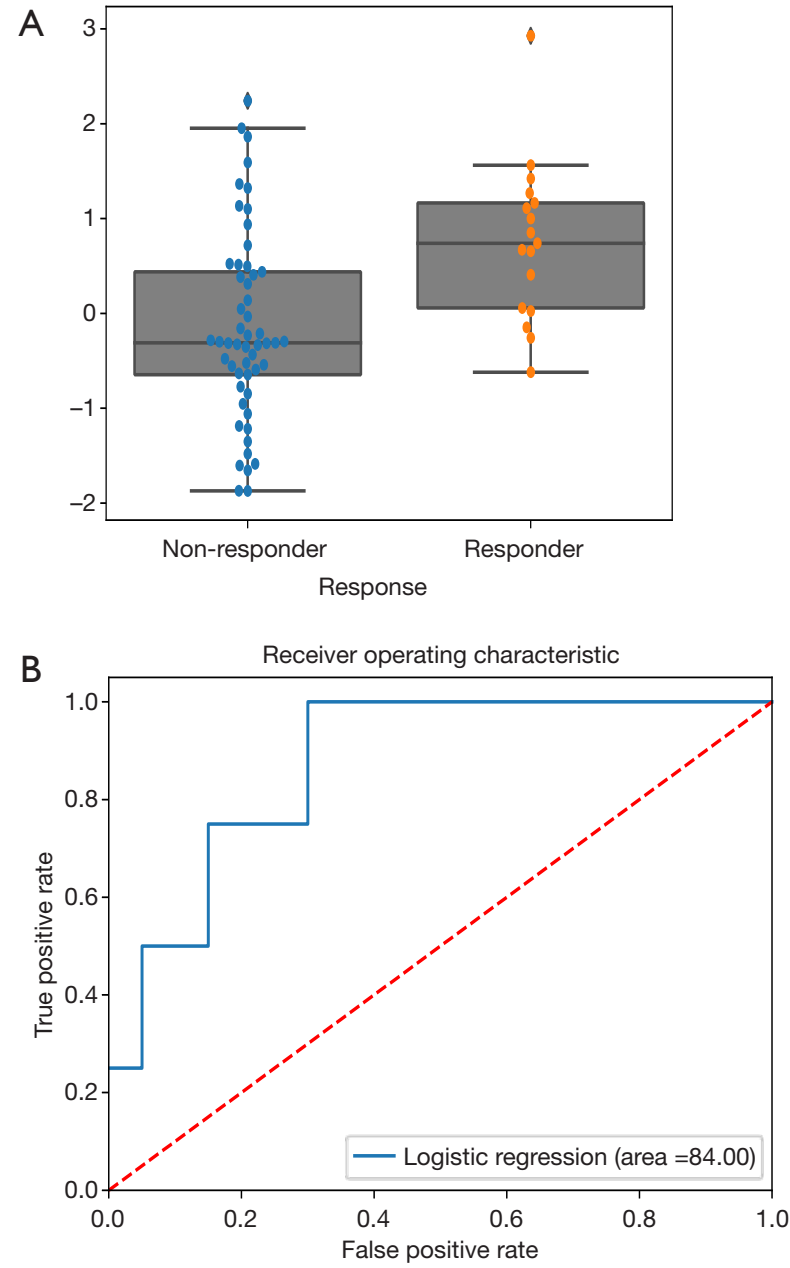

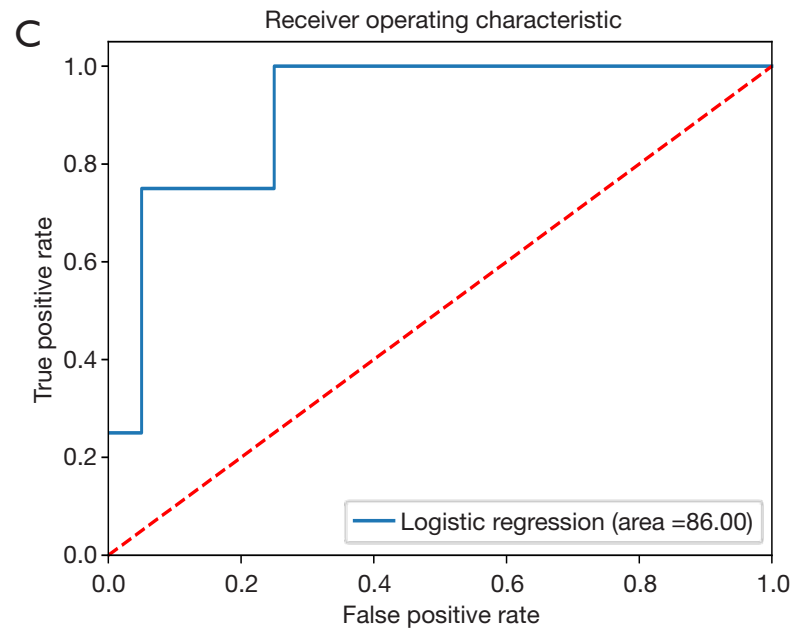

Figure 4 Predictive model for response at FU1. (A) Difference of radiomic value between responder and non-responder in terms of GLCM $^{\text {waveletHHL }}$. (B) Logistic regression model based on single radiomic feature. (C) Multivariate logistic recession model using selected radiomic features.

Goyal et al. conducted an analysis of size change for HCC and identified a significant $60 \%$ decrease in tumor volume at 3 months after SBRT (12). The average tumor response at FU1 in our study was -0.1789 , which approximately corresponded to $44.6 \%$ decreased in tumor volume $\left(0.8211^{3}\right)$. However, when tumors showing decrease in size were taken into account, about $67.9 \%$ decrease in volume was noted. Similar data were seen in lung cancer. Bhatt et al. showed a median $65 \%$ decrease (range, $12-96 \%$ ) in tumor volume from last SBRT in lung cancer with a median time from SBRT to FU1 of 88.5 days (11). The results suggested SBRT induces great tumor volume shrinkage. Nonetheless, the effect of SBRT in HCC could be influenced by various factors including liver functional parameters and radiation dose fractionation schedule (14). Kuo et al. used SBRT in HCC patients and higher local control rate was seen in smaller tumors (28). In addition, they found most complete response for tumors $\leq 4 \mathrm{~cm}$ occurred in $<3$ months after SBRT, and those tumors had sustained local control throughout the follow-up periods. In our study, 3 out of 5 complete responses (60\%) occurred at FU1 for tumors $<5 \mathrm{~cm}$, and all tumors achieving response at FU1 had sustained local control thereafter, paralleling their results. Moreover, the responders at FU1 tended to have higher survival probability, especially within 2 years. In our previous study, patients receiving SBRT had higher 2-year survival (9). These findings suggested that assessment at FU1 might help select the patients responsive to SBRT. 


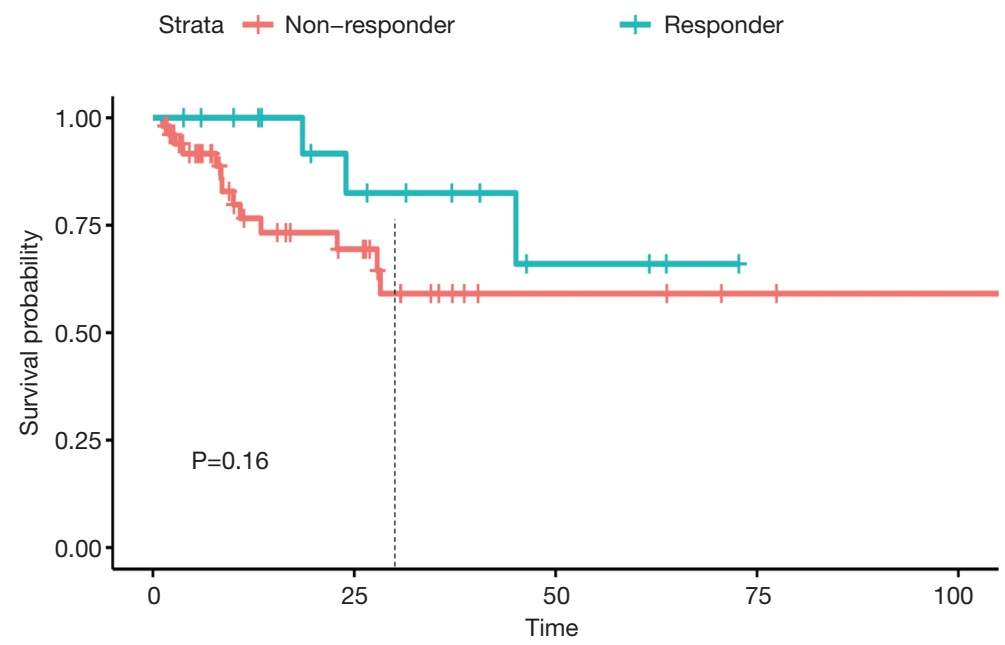

Figure 5 Kaplan-Meier survival analysis for tumors achieving response at FU1.

Depending on the tumor size and functional hepatic reserve, prescribed dose could range from 30 to $60 \mathrm{~Gy}$, with acceptable local control (29). In our study, we found no differences of tumor response among different ranges of EQD2, with or without tumor size normalization. However, significantly more responders were found at FU1 for tumors in the 'Norm-High' group. This indicated higher normalized EQD2 was more likely associated with tumor response at FU1, regardless of the size change. In addition, limited response seen in patients with $\mathrm{CP} \mathrm{B} / \mathrm{C}$ might also be contributed by lower EQD2.

According to the current evidence and our findings, predicting response at FU1 seems reasonable and clinically relevant. Currently, there had been no studies adopting radiomics to address the outcomes after SBRT in HCC. We explored the peritumoral region in HCC and established a predictive model with high performance based on the extracted features. The advantages of investigating the peritumoral regions included avoidance of the artifact generated from lipidol retention and correlation with immune therapy. For the later, Dai et al. had shown positive expression of programmed death ligand 1 in the peritumoral region is associated with worse overall and disease-free survival (23). Recently, Sun et al. proposed a peritumoral radiomic model with a $4 \mathrm{~mm}$ margin to predict immunotherapy response in solid tumors (30). This was especially crucial for SBRT, which has been shown to boost an abscopal response when combined with immunotherapy in various animal models (31). Ours adopted similar approaches and obtained a significant predictive feature $\left(\right.$ GLCM $\left.^{\text {waveletHHL }}\right)$ with high model accuracy $(0.83)$. This model was further improved by additional features with non-zero coefficients (accuracy $=0.88$ ), suggesting feasibility of peritumoral radiomics and its potential for immune response prediction.

This study was limited by its retrospective nature. Therefore, the follow-up time could not be standardized, making it impossible to track tumor response at a specific time point. Furthermore, because of missing information, some patients' response could not be evaluated. Lastly, the patient characteristics were not equally distributed. This unavoidably affects the statistical power of our study, which could be overcome by larger data set or prospective study.

\section{Conclusions}

The therapeutic impact of SBRT in HCC could be addressed by the tumor response at FU1, which corresponded to the local control about 1 year after therapy. Longer follow-ups are needed to further elucidate the radiation effect. On the other hand, this is the first study using radiomics to link the peritumoral region in HCC to the outcome after SBRT, opening the possibility of combined immunotherapy.

\section{Acknowledgments}

The authors thank the Cancer Registry Group of TriService General Hospital for the clinical data support. Funding: This study is partially supported by Kaohsiung 
Army Forces General Hospital (KAFGH_E_109048).

\section{Footnote}

Provenance and Peer Review: This article was commissioned by the Guest Editors (Chen-Hsi Hsieh, Hsin-Hua Nien, and Yu-Ming Huang) for the series "Precision Medicine for Cancer" published in Therapeutic Radiology and Oncology. The article has undergone external peer review.

Reporting Checklist: The authors have completed the STROBE reporting checklist. Available at http://dx.doi. org/10.21037/tro-20-38

Conflicts of Interest: All authors have completed the ICMJE uniform disclosure form (available at http://dx.doi. org/10.21037/tro-20-38). The series "Precision Medicine for Cancer" was commissioned by the editorial office without any funding or sponsorship. Hsing-Lung Chao serves as an unpaid editorial board member of Therapeutic Radiology and Oncology from May 2020 to Apr 2022. The authors have no other conflicts of interest to declare.

Ethical Statement: The authors are accountable for all aspects of the work in ensuring that questions related to the accuracy or integrity of any part of the work are appropriately investigated and resolved. This study was conducted in accordance with the Declaration of Helsinki (as revised in 2013). Because of the retrospective nature of this study, we have obtained approval from Institutional Review Board for a waiver of informed consent (IRB number: 1-107-05-016).

Open Access Statement: This is an Open Access article distributed in accordance with the Creative Commons Attribution-NonCommercial-NoDerivs 4.0 International License (CC BY-NC-ND 4.0), which permits the noncommercial replication and distribution of the article with the strict proviso that no changes or edits are made and the original work is properly cited (including links to both the formal publication through the relevant DOI and the license). See: https://creativecommons.org/licenses/by-nc-nd/4.0/.

\section{References}

1. Huynh E, Coroller TP, Narayan V, et al. CT-based radiomic analysis of stereotactic body radiation therapy patients with lung cancer. Radiother Oncol 2016;120:258-66.
2. Song SY, Choi W, Shin SS, et al. Fractionated stereotactic body radiation therapy for medically inoperable stage I lung cancer adjacent to central large bronchus. Lung Cancer 2009;66:89-93.

3. Siva S, Bressel M, Murphy DG, et al. Stereotactic Abative Body Radiotherapy (SABR) for Oligometastatic Prostate Cancer: A Prospective Clinical Trial. Eur Urol 2018;74:455-62.

4. Cárdenes HR, Price TR, Perkins SM, et al. Phase I feasibility trial of stereotactic body radiation therapy for primary hepatocellular carcinoma. Clin Transl Oncol 2010;12:218-25.

5. Andolino DL, Johnson CS, Maluccio M, et al. Stereotactic body radiotherapy for primary hepatocellular carcinoma. Int J Radiat Oncol Biol Phys 2011;81:e447-53.

6. Kang JK, Kim MS, Cho CK, et al. Stereotactic body radiation therapy for inoperable hepatocellular carcinoma as a local salvage treatment after incomplete transarterial chemoembolization. Cancer 2012;118:5424-31.

7. Bujold A, Massey CA, Kim JJ, et al. Sequential phase I and II trials of stereotactic body radiotherapy for locally advanced hepatocellular carcinoma. J Clin Oncol 2013;31:1631-9.

8. Moon DH, Wang AZ, Tepper JE. A prospective study of the safety and efficacy of liver stereotactic body radiotherapy in patients with and without prior liverdirected therapy. Radiother Oncol 2018;126:527-33.

9. Huang WY, Jen YM, Lee MS, et al. Stereotactic body radiation therapy in recurrent hepatocellular carcinoma. Int J Radiat Oncol Biol Phys 2012;84:355-61.

10. Yamashita H, Onishi H, Murakami N, et al. Survival outcomes after stereotactic body radiotherapy for 79 Japanese patients with hepatocellular carcinoma. J Radiat Res 2015;56:561-7.

11. Bhatt AD, El-Ghamry MN, Dunlap NE, et al. Tumor volume change with stereotactic body radiotherapy (SBRT) for early-stage lung cancer: evaluating the potential for adaptive SBRT. Am J Clin Oncol 2015;38:41-6.

12. Goyal K, Einstein D, Yao M, et al. Cyberknife stereotactic body radiation therapy for nonresectable tumors of the liver: preliminary results. HPB Surg 2010;2010:309780.

13. Lu LC, Hsu CH, Hsu C, et al. Tumor Heterogeneity in Hepatocellular Carcinoma: Facing the Challenges. Liver Cancer 2016;5:128-38.

14. Schaub SK, Hartvigson PE, Lock MI, et al. Stereotactic Body Radiation Therapy for Hepatocellular Carcinoma: Current Trends and Controversies. Technol Cancer Res Treat 2018;17:1533033818790217. 
15. Lambin P, van Stiphout RG, Starmans $M H$, et al. Predicting outcomes in radiation oncology--multifactorial decision support systems. Nat Rev Clin Oncol 2013;10:27-40.

16. Aerts HJ, Velazquez ER, Leijenaar RT, et al. Decoding tumour phenotype by noninvasive imaging using a quantitative radiomics approach. Nat Commun 2014;5:4006.

17. Raman SP, Schroeder JL, Huang P, et al. Preliminary data using computed tomography texture analysis for the classification of hypervascular liver lesions: generation of a predictive model on the basis of quantitative spatial frequency measurements--a work in progress. J Comput Assist Tomogr 2015;39:383-95.

18. Li M, Fu S, Zhu Y, et al. Computed tomography texture analysis to facilitate therapeutic decision making in hepatocellular carcinoma. Oncotarget 2016;7:13248-59.

19. Cozzi L, Dinapoli N, Fogliata A, et al. Radiomics based analysis to predict local control and survival in hepatocellular carcinoma patients treated with volumetric modulated arc therapy. BMC Cancer 2017;17:829.

20. Grudé P, Conti F, Mennecier D, et al. MDR1 gene expression in hepatocellular carcinoma and the peritumoral liver of patients with and without cirrhosis. Cancer Lett 2002;186:107-13.

21. Zhu XD, Zhang JB, Zhuang PY, et al. High expression of macrophage colony-stimulating factor in peritumoral liver tissue is associated with poor survival after curative resection of hepatocellular carcinoma. J Clin Oncol 2008;26:2707-16.

22. Ju MJ, Qiu SJ, Fan J, et al. Peritumoral activated hepatic stellate cells predict poor clinical outcome in hepatocellular carcinoma after curative resection. Am J Clin Pathol 2009;131:498-510.

23. Dai X, Xue J, Hu J, et al. Positive Expression of

doi: $10.21037 /$ tro-20-38

Cite this article as: Dai YH, Shen PC, Wang YF, Chang WC, Lo CH, Yang JF, Lin CS, Chao HL, Lu CS, Huang WY. Evaluation and prediction of therapeutic response for patients with hepatocellular carcinoma receiving stereotactic body radiotherapy using serial computed tomography scans and radiomics. Ther Radiol Oncol 2020;4:29.
Programmed Death Ligand 1 in Peritumoral Liver Tissue is Associated with Poor Survival after Curative Resection of Hepatocellular Carcinoma. Transl Oncol 2017;10:511-7.

24. Lo CH, Liu MY, Lee MS, et al. Comparison Between Child-Turcotte-Pugh and Albumin-Bilirubin Scores in Assessing the Prognosis of Hepatocellular Carcinoma After Stereotactic Ablative Radiation Therapy. Int J Radiat Oncol Biol Phys 2017;99:145-52.

25. Llovet JM, Di Bisceglie AM, Bruix J, et al. Design and endpoints of clinical trials in hepatocellular carcinoma. J Natl Cancer Inst 2008;100:698-711.

26. Lencioni R, Llovet JM. Modified RECIST (mRECIST) assessment for hepatocellular carcinoma. Semin Liver Dis 2010;30:52-60.

27. Hui Zou TH. Regularization and variable selection via the elastic net. Journal of the Royal Statistical Society, Series B 2005;67: 301-20.

28. Kuo HT, Que J, Lin LC, et al. Impact of tumor size on outcome after stereotactic body radiation therapy for inoperable hepatocellular carcinoma. Medicine (Baltimore) 2017;96:e9249.

29. Wang PM, Chung NN, Hsu WC, et al. Stereotactic body radiation therapy in hepatocellular carcinoma: Optimal treatment strategies based on liver segmentation and functional hepatic reserve. Rep Pract Oncol Radiother 2015;20:417-24.

30. Sun R, Limkin EJ, Vakalopoulou M, et al. A radiomics approach to assess tumour-infiltrating CD8 cells and response to anti-PD-1 or anti-PD-L1 immunotherapy: an imaging biomarker, retrospective multicohort study. Lancet Oncol 2018;19:1180-91.

31. Ngwa W, Irabor OC, Schoenfeld JD, et al. Using immunotherapy to boost the abscopal effect. Nat Rev Cancer 2018;18:313-22. 